\title{
Small Cell Lung Cancer Invading the Left Atrium With Subsequent Malignant Embolic Stroke: A Case Report and Review of Literature
}

\author{
Raghav Chandra ${ }^{\mathrm{a}, \mathrm{d}}$, Ashraf Abugroun ${ }^{\mathrm{b}}$, Alan Goldberga ${ }^{\mathrm{a}}$, Elizabeth Cooney ${ }^{\mathrm{a}}$, \\ Swati Mehrotra ${ }^{\mathrm{c}}$, Annabelle Volgman ${ }^{\mathrm{a}}$
}

\begin{abstract}
Cardiac tumors are uncommon, and the vast majority of them are metastases from extracardiac sources. Metastatic spread to the heart causes symptoms by mechanical obstruction of circulation, direct myocardial invasion, or distal embolization. We herein report a case of a 58-year-old male who presented to the hospital with multilobar intracranial embolic infarcts who was found to have small cell lung cancer (SCLC) with invasion of the left atrium and pulmonary artery resulting in malignant embolic stroke. Cerebral tumor thromboembolism from SCLC is extremely rare. This case demonstrates the thromboembolic risk associated with metastatic endoluminal cardiac tumors.
\end{abstract}

Keywords: Small-cell lung cancer; Heart; Embolism

\section{Introduction}

Cardiac tumors are rare and poorly understood entities. The vast majority of these are metastases from external malignancies including lung cancer, esophageal cancer, lymphoma, melanoma and sarcoma, with primary cardiac tumors being exceptionally rare and usually benign (i.e. myxoma) $[1,2]$. Extracardiac primary tumors can spread to the heart through direct extension, lymphatic, or hematogenous spread. Metastases may involve any cardiac structure, but the pericardium is most often involved and the endocardium the least $[1,2]$. Endoluminal cardiac tumors may pose a significant thromboembolic risk including stroke and systemic arterial embolism. Here, we report a case of a 58 -year-old male who presented to

Manuscript submitted June 23, 2018, accepted September 22, 2018

${ }^{a}$ Rush Medical College, Chicago, IL, USA

bAdvocate Illinois Masonic Medical Center, Chicago, IL, USA

'Department of Pathology, Stritch School of Medicine of Loyola University, Maywood, IL 60153, USA

${ }^{\mathrm{d} C o r r e s p o n d i n g ~ A u t h o r: ~ R a g h a v ~ C h a n d r a, ~ R u s h ~ M e d i c a l ~ C o l l e g e, ~ C h i c a g o, ~}$

IL, USA. Email: chandraraghav92@gmail.com

doi: https://doi.org/10.14740/cr752w the hospital with multilobar intracranial embolic infarcts with primary origin from primary small cell lung cancer (SCLC) arising from the left upper lobe of the lung and invading the left atrium and pulmonary artery.

\section{Case Report}

A 58-year-old male with a history of well-controlled hypertension, chronic smoking, and recently diagnosed biopsy-confirmed small cell lung cancer (SCLC) of the left upper lobe presented to the hospital with a left field cut, right gaze palsy, and left hand weakness (Fig. 1). He had previously been admitted to an outside hospital after developing sudden-onset tonicclonic seizures and expressive aphasia. A magnetic resonance imaging (MRI) scan of the brain at that time revealed scattered subcentimeter ischemic infarcts in the right and left frontal lobes, right parietal lobe, and the left cerebellar hemisphere. He was transferred to our institution for further management.

Upon admission, the patient was admitted to the neurosciences intensive care unit for further monitoring. He was placed on continuous electroencephalographic monitoring. Computed tomography (CT) of the brain showed new mild hypodensities in the right subcortical frontal lobe with stable hypodensities in the periventricular white matter, left subcortical frontal and left occipital lobes (Fig. 2). A transthoracic echocardiogram (TTE) revealed a moderately dilated left atrium with a $40 \times 39 \mathrm{~mm}$ spherical echogenic, heterogeneous mass originating from a left pulmonary vein (Figs. 3, 4). Given that the patient had no significant cardiovascular history, it was concluded that the embolic infarcts were likely secondary to tumor shower from the left atrial metastasis from his primary SCLC. The patient was scheduled to undergo cardiac MRI imaging to further characterize the left atrial lesion and extent of invasion, but given the risk of intubation and sedation, this was deferred. A CT-angiogram and chest X-ray of the chest was conducted which demonstrated encasement of the left main pulmonary artery with direct extension of the left pulmonary vein and left atrium (Figs. 5, 6).

In the setting of metastatic left atrial invasion with metastatic thromboembolic spread to the brain, the patient was started on anticoagulation with a heparin drip in the hospital and later transitioned to enoxaparin. His neurologic status improved and he was transferred to the oncology service 

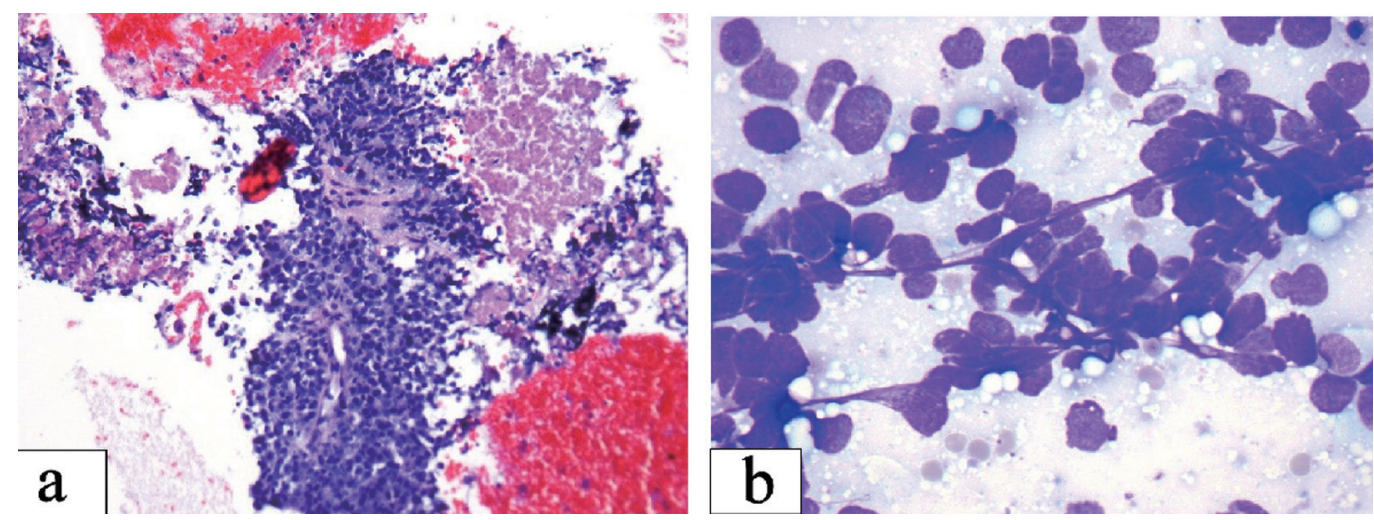

Figure 1. (a) Small cell carcinoma: loosely cohesive clusters of small cells with high nuclear cytoplasmic ratio and fine chromatin. Streaks of nuclear material and nuclear molding as seen are important diagnostic clue (Smear, Diff-Quik stain, $\times 600$ ). (b) Necrosis seen as granular pink material in the upper right corner may be prominent. The center of the field shows preserved tumor cells with high nuclear cytoplasmic ratio around capillaries (Cell block section, H\&E stain, × 200).

for initiation of chemotherapy. After initiation, he developed atrial fibrillation with rapid ventricular response that was subsequently controlled with metoprolol. He was discharged in stable condition from the hospital and has been following up with his oncologist and cardio-oncologist for continued man-

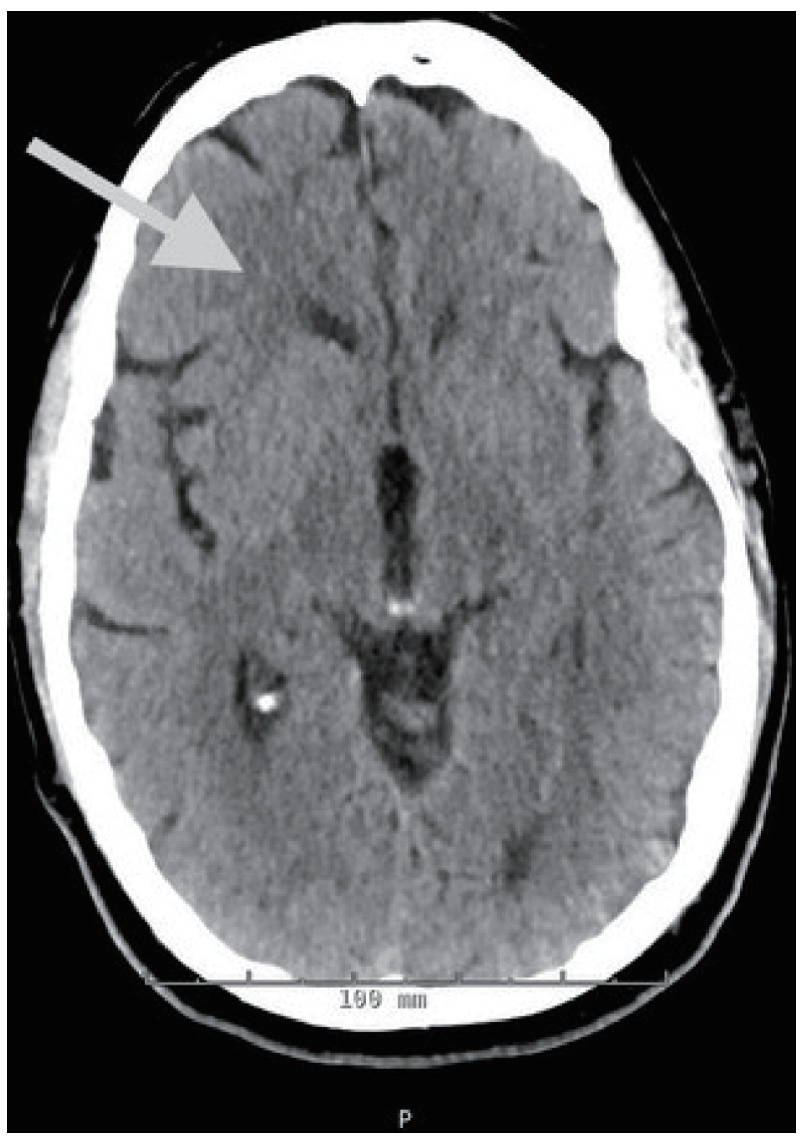

Figure 2. CT imaging of the brain demonstrating new mild hypodensities in the right subcortical frontal lobe, stable milder hypodensities in the left subcortical frontal lobe, stable hypodensities in the left occipital lobe, and minimal periventricular white matter hypodensities. agement of SCLC.

\section{Discussion}

Cardiac metastases are uncommonly reported but may be present in up to $18.3 \%$ of all patients with malignant disease [1]. They often portend a poor prognosis. Almost any primary tumor can cause cardiac metastasis, but common sources include melanomas, lymphomas, breast cancers, and lung cancers [1, 3]. While cardiac invasion can be initially asymptomatic, they can cause life-threatening complications including arrhythmias, atrioventricular nodal block, pericardial effusions, myocardial infarction, and pulmonary embolism [2]. In this report, we present a case of SCLC invading into the left atrium with resulting malignant embolic stroke.

SCLC is a highly aggressive and often fatal pulmonary malignancy with rapid intrathoracic growth, early metastasis, and multiple paraneoplastic syndromes. It is strongly associated with smoking and accounts for $14 \%$ of all lung cancer cases [4]. The most frequent sites of metastasis include mediastinal lymph nodes, liver, bone, adrenals, and brain. Cardiac involvement is uncommon and therefore poorly documented in the literature, with the exception of isolated case reports $[2,5]$.

Cerebral tumor thromboembolism from SCLC is extremely rare and to our knowledge, only one other case report demonstrated a similar presentation of SCLC with left atrial involvement [5]. In general, left atrial invasion from lung cancer is uncommon, but well-documented in the literature and may occur in up to $10 \%$ of lung cancer cases [6]. There are isolated cases of non-small cell lung cancer (NSCLC) with left atrial invasion resulting in multifocal cerebral infarcts [7, 8]. Systemic tumor embolism from left atrial involvement has also been documented from other malignancies including chondrosarcoma with left atrial involvement resulting in thromboembolism of the left common iliac artery, pulmonary embolism due to right ventricular metastasis from squamous cell carcinoma of the cervix, as well as others [9-11]. Tumor emboli from cardiac involvement uniformly portend a grave prognosis, and treatment for these patients is usually palliative. In particular, 


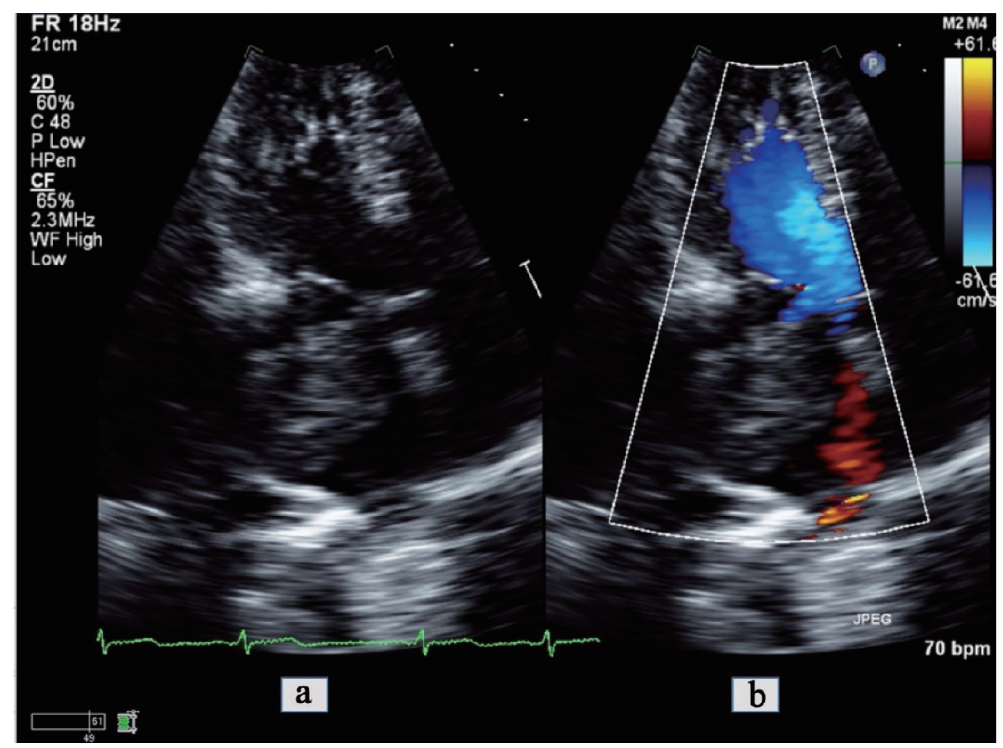

Figure 3. Apical 4-chamber view transthoracic echocardiogram zoomed in on the left atrium (a) with color comparison (b) showing a large tumor invading into the left atrial cavity.

SCLC, regardless of cardiac involvement, is usually too far advanced for surgical resection and the mainstay of treatment is chemotherapy and radiation.

Given its rare incidence, no clear guidelines have been established for diagnosis of cardiac metastasis. All patients with suspected cerebral tumor emboli from cardiac invasion should undergo standard acute stroke workup including head CT and MRI scan, chest X-ray, transthoracic echocardiography, and CT imaging of the chest. If cardiac involvement is noted on echocardiography or CT imaging, cardiac MRI may be useful in better characterizing structural involvement of the mass. Orcurto et al suggested that PET-CT imaging may be useful for the assessment of equivocal cardiac lesions found on other imaging modalities [2]. As pericardial involvement is most common, further evaluation may reveal elevated central venous pressure, pulsus paradoxus, low voltage QRS on electrocardiography, and possible tamponade. Pericardiocentesis

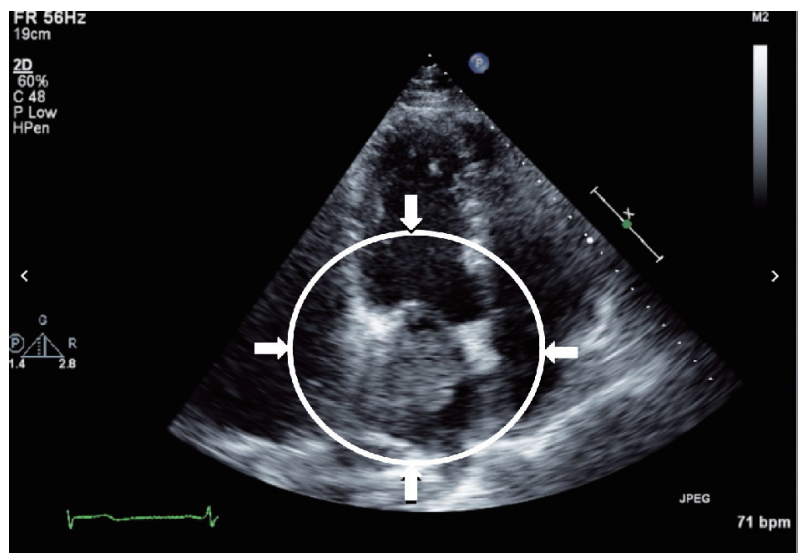

Figure 4. Transthoracic echocardiogram demonstrating infiltrating mass in the left atrium with possible extension through the mitral valve into the left ventricle. often reveals hemorrhagic pericardial effusion with positive cytology [1]. Patients with diffuse cardiac involvement may present in heart failure or myocardial infarction from tumor embolism or invasion into the coronary vessels [1].

Management of patients with SCLC with left atrial involvement is primarily nonsurgical and usually palliative with chemotherapy and radiation. Interventions for acute cardiac complications, including arrhythmia, pericarditis, tamponade, or infarction should be initiated. Our patient began chemotherapy for symptomatic relief. There is some role for surgical resection of lung cancer with left atrial involvement without evidence of systemic disease. In a review of 15 patients with NSCLC with left atrial involvement who underwent extended pneumonectomy with left atrial resection without cardiopul-

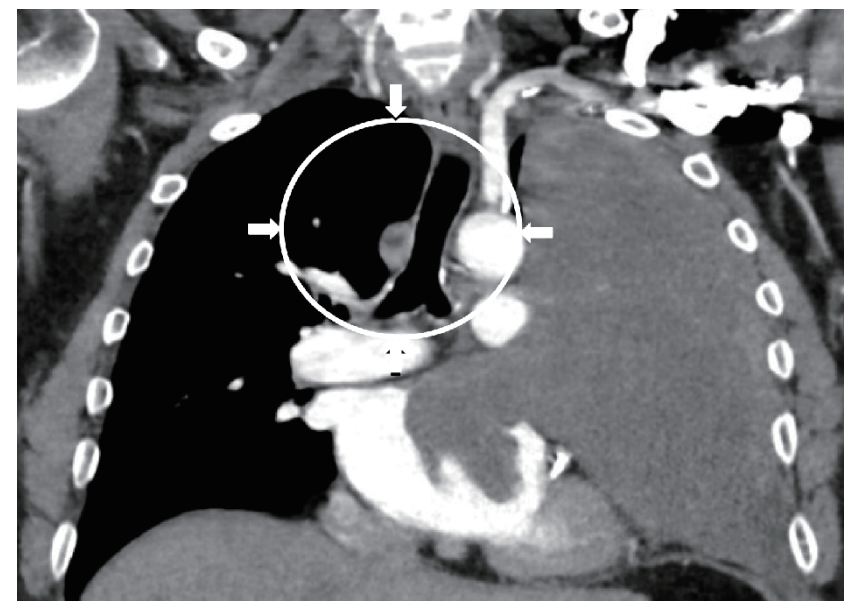

Figure 5. CT imaging with contrast of the chest demonstrating the large soft tissue map entirely displacing the left upper lobe of the lung with encasement of the left main pulmonary artery and invasion of both the left upper anterior pulmonary vein and left atrial cavity. The lesion abuts the left aortic arch, main pulmonary trunk, and the left cardiac border. 

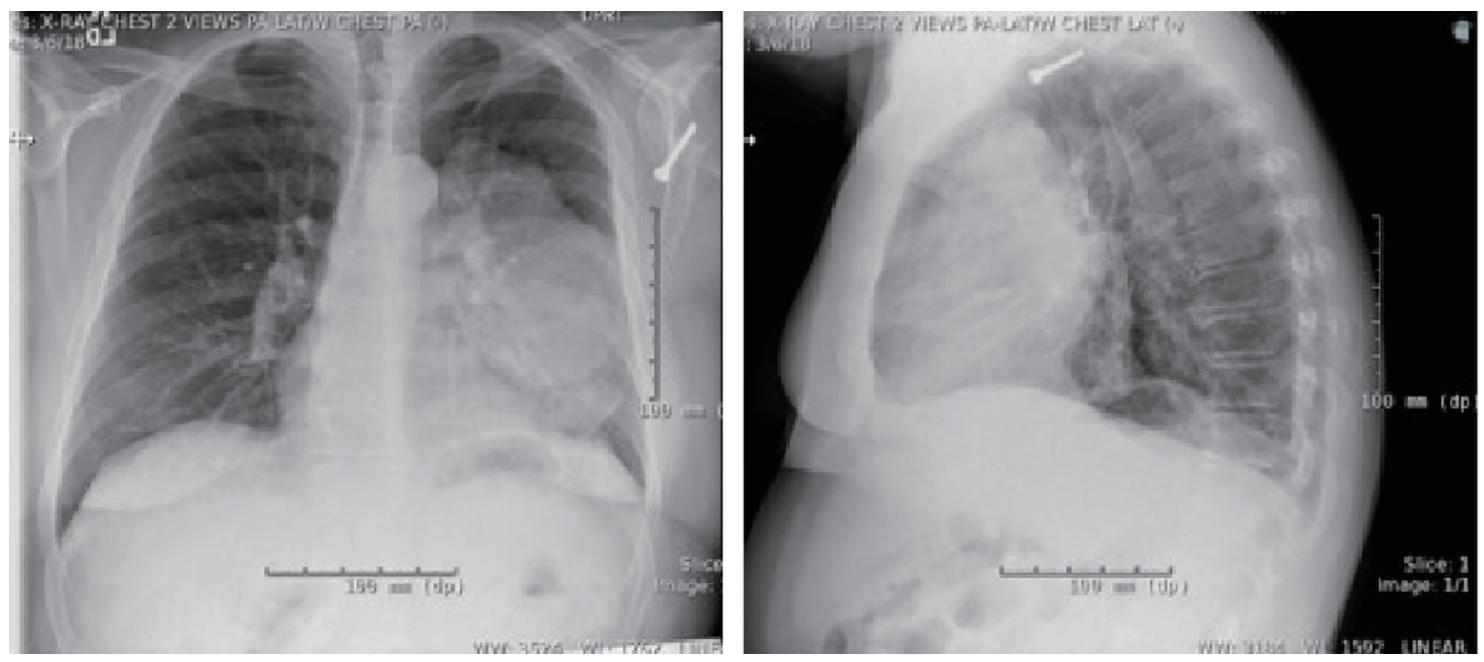

Figure 6. Upright frontal and lateral X-rays demonstrating large, left-sided hilar mass.

monary bypass, all patients were able to achieve a negative margin and the overall 3-year survival was 39\% [12]. The authors selected such patients based on the exclusion of mediastinal invasion or involvement of regional lymph nodes on mediastinoscopy. They also recommended that most of these patients undergo induction chemotherapy [12]. Other case series have reported similar results after surgical resection both with and without cardiopulmonary bypass as well $[13,14]$. It is important to note that there is scant evidence of attempting surgical resection if the primary tumor was SCLC, which is significantly more aggressive in its tumor biology. In one case series of 12 patients who underwent left atrial resection in conjunction with pneumonectomy, one patient had a combined epidermoid and SCLC in whom surgical resection was attempted. To our knowledge, such resection has not been attempted in patients with pure SCLC, as is the case for our patient [13]. Furthermore, such aggressive surgical intervention is clearly contraindicated in patients with evidence of systemic disease, as is most likely the case in our patient.

\section{Conclusions}

SCLC has a potential for invasion of the heart. Cardiac invasion by the tumor can be complicated with development of multifocal embolic cerebral infarcts. Given the aggressive nature of the SCLC, treatment is mainly palliative through chemotherapy with limited role for surgical intervention.

\section{Acknowledgments}

None to declare.

\section{Financial Disclosure}

None to disclose.

\section{Conflict of Interest}

There is no conflict of interest to declare.

\section{Informed Consent}

Informed consent was verbally obtained.

\section{Author Contributions}

Raghav Chandra: manuscript composition, editing, and literature review; Ashraf Abugroun: manuscript composition, literature review, and manuscript editing; Alan Goldberg: manuscript editing and literature review; Elizabeth Cooney: manuscript editing; Swati Mehrotra: pathologic analysis and description; Annabelle Volgman: manuscript editing, literature review, figure analysis and captions.

\section{References}

1. Bussani R, De-Giorgio F, Abbate A, Silvestri F. Cardiac metastases. J Clin Pathol. 2007;60(1):27-34.

2. Orcurto MV, Delaloye AB, Letovanec I, Martins Favre M, Prior JO. Detection of an asymptomatic right-ventricle cardiac metastasis from a small-cell lung cancer by F-18-FDG PET/CT. J Thorac Oncol. 2009;4(1):127-130.

3. Paraskevaidis IA, Michalakeas CA, Papadopoulos $\mathrm{CH}$, Anastasiou-Nana M. Cardiac tumors. ISRN Oncol. 2011;2011:208929.

4. Jackman DM, Johnson BE. Small-cell lung cancer. Lancet. 2005;366(9494):1385-1396.

5. Duncan MD, Swinburne AJ, Sahni S, Zuckerman JE, Hacobian M. Small cell lung cancer presenting as a cardiac mass with embolic phenomena. Am J Med. 2017;130(2):e55-e57. 
6. Ma Q, Liu D, Liu P, Chen J, Xie Z, D'Amico TA. Extensive invasion of the left atrium by lung cancer. Ann Thorac Surg. 2013;96(2):685-687.

7. Park JH, Seo HS, Park SK, Suh J, Kim DH, Cho YH, Lee NH. Spontaneous systemic tumor embolism caused by tumor invasion of pulmonary vein in a patient with advanced lung cancer. J Cardiovasc Ultrasound. 2010;18(4):148-150.

8. Navi BB, Kawaguchi K, Hriljac I, Lavi E, DeAngelis LM, Jamieson DG. Multifocal stroke from tumor emboli. Arch Neurol. 2009;66(9):1174-1175.

9. Boland TW, Winga ER, Kalfayan B. Chondrosarcoma: a case report with left atrial involvement and systemic embolization. J Thorac Cardiovasc Surg. 1977;74(2):268-272.

10. Itoh K, Matsubara T, Yanagisawa K, Hibi N, Nishimura $\mathrm{K}$, Kambe T, Sakamoto N, et al. Right ventricular metas- tasis of cervical squamous cell carcinoma. Am Heart J. 1984;108(5):1369-1371.

11. Schreffler SM, Paolo WF, Kloss BT. Spontaneous showering of tumor emboli in a patient with advanced primary lung cancer: a case report. Int J Emerg Med. 2012;5(1):27.

12. Spaggiari L, M DA, Veronesi G, Pelosi G, de Pas T, Catalano G, de Braud F. Extended pneumonectomy with partial resection of the left atrium, without cardiopulmonary bypass, for lung cancer. Ann Thorac Surg. 2005;79(1):234-240.

13. Shirakusa T, Kimura M. Partial atrial resection in advanced lung carcinoma with and without cardiopulmonary bypass. Thorax. 1991;46(7):484-487.

14. Ferguson ER, Jr., Reardon MJ. Atrial resection in advanced lung carcinoma under total cardiopulmonary bypass. Tex Heart Inst J. 2000;27(2):110-112. 